Rapid Reviews COVID-19•

\title{
Review 2: "SARS-CoV-2 Aerosol Transmission in Schools: The Effectiveness of Different Interventions"
}

Richard M. Lynch, Ph.D. ${ }^{1}$

${ }^{1}$ Preferred Management Corporation, President, UNITED STATES

Published on: Sep 19, 2021

License: Creative Commons Attribution 4.0 International License (CC-BY 4.0). 


\section{$\underline{\text { RR:C19 Evidence Scale rating by reviewer: }}$}

- Reliable. The main study claims are generally justified by its methods and data. The results and conclusions are likely to be similar to the hypothetical ideal study. There are some minor caveats or limitations, but they would/do not change the major claims of the study. The study provides sufficient strength of evidence on its own that its main claims should be considered actionable, with some room for future revision.

$* * * * * * * * * * * * * * * * * * * * * * * * * * * * * * * * * * * * * * *$

\section{Review:}

The manuscript "SARS-CoV-2 Aerosol Transmission in Schools: the Effectiveness of Different Interventions" describes the results of modelling SARS-Co-V-2 virion exposure dose within classrooms using the COVID Airborne Risk Assessment (CARA) tool, assuming 1 infected person standing within the room over the course of a school day. The interventions considered include 1) open windows, 2) installation of portable HEPA filters, and 3) use of masks on occupants. Reductions in virion accumulation rates under combinations of these intervention methods are compared to estimated virion accumulations in an unventilated comparison classroom scenario. The authors report that opening 6 classroom windows generates a 14-fold reduction in exposure during winter and a 2-fold reduction during spring/summer; approximately the same as opening two windows fully during winter. Masks use results in an 8-fold reduction. HEPA filters in combination with partially opened windows yielded a 2.5-to-4-fold reduction. All interventions combined resulted in an approximate 30 -fold decrease. The premise of the modelling and conclusions are, in my opinion appropriate, important, and the article is worthy of publication with the following clarifications, which in my view are necessary for practical consideration and application by readers:

1. The assumptions within the modelling are that the classrooms have no mechanical ventilation, but are instead ventilated solely by windows along one side. This is true in many older school buildings, as well as converted church-to school buildings throughout the U.S., making this a valid and important subject for study. Therefore, the article should specify that this modelling was for naturally ventilated classrooms, not mechanically ventilated ones. I recommend that the title be revised to address this. The revised tittle for consideration is "SARS-CoV-2 Aerosol Transmission in Schools: the Effectiveness of Different Interventions in Naturally Ventilated Classrooms." 
2. The above, notwithstanding, many schools have mechanical ventilation which may include unit ventilators along the window wall, or overhead HVAC supply and return registers serviced by rooftop air handling units. It is our experience in assessing hundreds of schools and classrooms throughout New Jersey, New York and Pennsylvania, that total airflow and outdoor air introduction rates from these mechanical ventilation systems vary greatly dependent upon factors such as design specifications, vintage, state of repair, filters installed, thermostat programming and other factors.

3. For these mechanical ventilation systems, the outdoor air introduction rates may vary from 0 to $100 \%$ depending on condition, thermostat settings, outdoor air temperature linkage position etc. Pre-COVID these were often set to a minimum outdoor air introduction rate of 10-15\%, and many are now being increased to comply with recent CDC and ASHRAE COVID recommendations. Most mechanical ventilation systems have modest filter efficiencies, (MERV 8-11 at best).

4. Based upon points 2 and 3 above, and because the authors use the difference in outdoor versus indoor temperatures $(\sqrt{ } \Delta \mathrm{T})$ as the driving factor for outdoor air introduction rates via windows, I suggest that the authors report the outdoor air introduction rates in (cubic feet per minute (cfm) as well as in Air Changes per Hour $(\mathrm{ACH})$ computed by the CARA model under each of the winter versus spring/summer seasons (under both the 6 windows fully open as well as 2 windows partially open scenarios) so that readers can apply the implications of the findings to conditions in naturally and mechanically ventilated classrooms as described in discussion point \#5 below.

5. The discussion of $\mathrm{CO} 2$ as a measure of natural ventilation is based upon the ASHRAE 62 guidelines which indicate bio-effluents within occupied buildings are diluted to acceptable ranges when outdoor air introduction rates exceed approximately 15 cubic feet per minute per person. So for a $160 \mathrm{~m}^{\wedge} 3$ typical classroom servicing as an example 20-25 persons, outdoor air introduction rates of approximately 300-375 cubic feet per minute ( $\mathrm{cfm}$ ) would be recommended as the minimum outdoor introduction rate. At steady state equilibrium conditions, this level of outdoor air introduction would be expected to result in indoor $\mathrm{CO} 2$ concentrations of less than 1,000 parts per million (assuming outdoor air contained approximately 300 ppm $\mathrm{CO} 2$ ). While the underlying equations that yield these results are beyond the scope of this paper, the authors should make the link between indoor $\mathrm{CO} 2$ and ventilation rates clear. 


\begin{abstract}
All combined, it is my professional opinion that these clarifications will help readers better; 1) understand the implications of the study, 2) applicability of the study to naturally ventilated classrooms, 3) applicability to mechanically ventilated classrooms, and 4) steps which can be taken to attenuate COVID-19 transmission risks when mechanically ventilation systems fail due to broken parts, failed motors or when fresh air dampers are closed.
\end{abstract}

References: 1.Assess Ventilation When Determining Safe Distancing in Schools to Control Coronavirus Disease 2019 (COVID-19) Transmission Richard M Lynch, Elissa Favata, Michael Gochfeld Clinical Infectious Diseases, ciab353, https://doi.org/10.1093/cid/ciab353 